\title{
Doença de Forestier
}

\section{Relato de caso}

\author{
Fernando Luíz Rolemberg Dantas*, Antônio Carlos Vieira Caires*, \\ Ana Lúcia Vaz de Melo**, Jair Leopoldo Raso* \\ Serviços de Neurocirurgia e Reumatologia do Hospital BIOCOR. Belo Horizonte, MG
}

\begin{abstract}
RESUMO
A doença de Forestier, ou hiperostose esquelética idiopática difusa (diffuse idiopatic skeletal hyperostosis) é uma doença sistêmica de etiologia desconhecida caracterizada pela presença de calcificação e ossificação da face ântero-lateral de no mínimo quatro corpos vertebrais adjacentes, relativa preservação da altura dos discos intervertebrais dos segmentos envolvidos e grandes osteófitos. Os autores apresentam um caso de um paciente do sexo masculino de 55 anos de idade com quadro de disfagia conseqüente a essa doença.

Discutem no presente registro os aspectos clínicos da doença, o tratamento e o diagnóstico diferencial.
\end{abstract}

\section{PALAVRAS-CHAVE}

Hiperostose esquelética idiopática difusa. Doença de Forestier.

\begin{abstract}
Forestier's disease. Case report associated with the development of important ostheophyts. presentation, the differential diagnosis and the treatment are discussed.

\section{KEYWORDS}

Diffuse idiopathic skeletal hyperostosis. Forestier's disease.
\end{abstract}

Forestier disease or diffuse idiopathic skeletal hyperostosis is a systemic abnormality of unknown etiology, that presents with calcification and ossification along anterolateral aspect of four contiguos vertebral bodies with relative preservation of intervertebral disc height in affected areas and

The authors present a case of a 55 year-old man, complaining of dysphagia. The clinical

\section{Introdução}

A doença de Forestier, ou hiperostose esquelética idiopática difusa, é uma afecção relativamente freqüente, atingindo cerca de $10 \%$ a $20 \%$ da população acima de 70 anos $^{8,33}$, sendo encontrada em $6 \%$ a $12 \%$ em séries de autópsias, acometendo mais comumente pacientes do sexo masculino acima de $50 \operatorname{anos}^{25,35}$. Geralmente é uma doença assintomática. Contudo, disfagia, dispnéia, estridor laríngeo, compressão medular, compressão radicular, compressão de nervos periféricos, pneumonia de aspiração têm sido relatados em associação com essa afecção $0^{1,5,6,9,10,17,19,21,22,24,27,28,30,32}$. Leroux e cols. ${ }^{15}$, estudando 100 pacientes com estenose adquirida do canal lombar, encontraram $46 \%$ dos pacientes com doença de Forestier ${ }^{15}$.
Em razão de sua raridade no meio neurocirúrgico, relatamos um caso da doença salientando os achados clínicos e o diagnóstico diferencial.

\section{Relato do caso}

Paciente do sexo masculino, 55 anos de idade, relatava disfagia de cerca de 6 meses de duração, inicialmente aos alimentos sólidos, progredindo para a dificuldade de deglutição de líquidos.

Ao exame, constatou-se apenas diminuição generalizada dos movimentos da coluna, sem rigidez. A radiografia simples da coluna cervical revelou a presença de osteófitos muito desenvolvidos envol-

* Neurocirurgião.

** Reumatologista. 
vendo os corpos vertebrais de $\mathrm{C} 2$ a $\mathrm{C} 6$, principalmente em C4-C5 (Figura 1). O esofagograma demonstrou deslocamento para frente e para a direita do esôfago e traquéia, com compressão mais evidente ao nível C4C5 pelos osteófitos (Figura 2). A tomografia da coluna cervical revelou ossificação do ligamento longitudinal anterior na região cervical de C2-C6 (Figura 3).

Radiografias da coluna torácica, lombar e sacral mostraram calcificação e ossificação do ligamento longitudinal anterior.

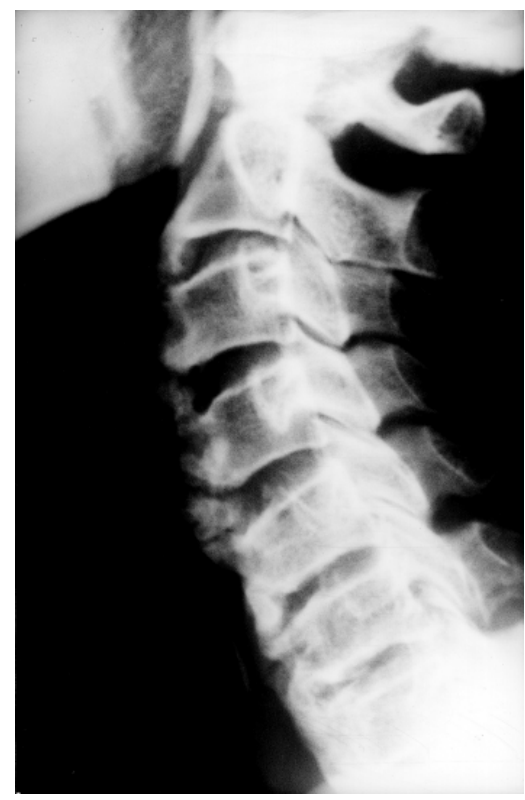

Figura 1 - Rx da coluna cervical. Osteófitos envolvendo os corpos vertebrais de $C 2$ a C6, principalmente em C4-C5.

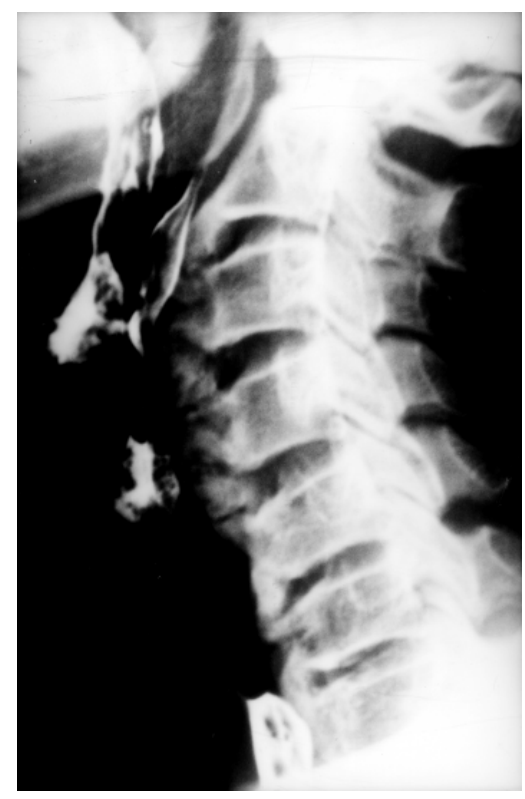

Figura 2 - Esofagograma. Deslocamento anterior do esôfago e da traquéia com compressão mais evidente ao nível C4-C5.

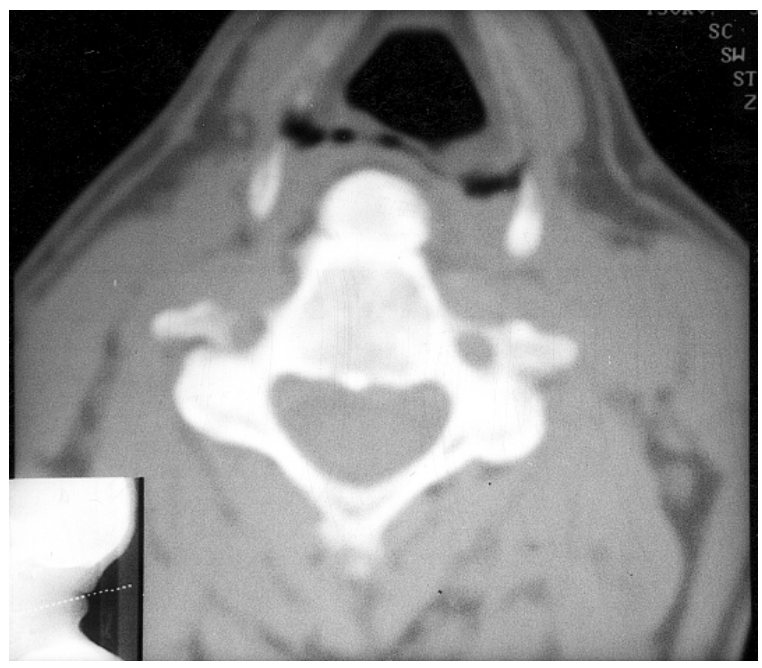

Figura 3 - Tomografia. Ossificação do ligamento longitudinal anterior.

O paciente foi submetido, sob anestesia geral, a uma cervicotomia anterior, expondo-se desde $\mathrm{C} 2$ a $\mathrm{C} 7$, e os osteófitos de $\mathrm{C} 2$ a $\mathrm{C} 6$ foram removidos com goiva e drill. Não foi empregado enxerto. O esôfago estava mais aderido no nível C4-C5, onde existia o maior osteófito.

O paciente evoluiu bem no pós-operatório, sem complicações, com melhora gradativa da disfagia já a partir do segundo dia, ficando praticamente assintomático em cerca de uma semana.

A radiografia de controle feita no pós-operatório imediato demonstrou boa ressecção (Figura 4). O exame anatomopatológico dos fragmentos removidos demonstrou tecido osteocartilaginoso com área recoberta por pannus e fibrose no tecido ósseo subcondral.

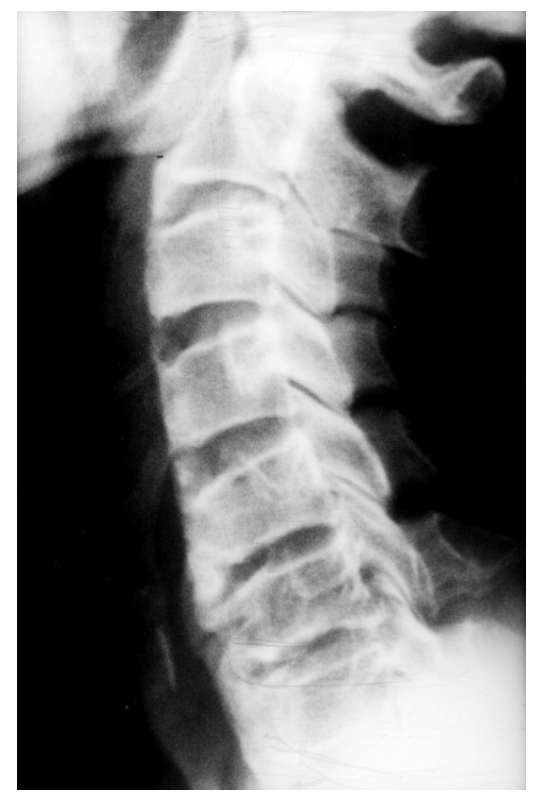

Figura $4-R x$ da coluna cervical. Pós-operatório imediato demonstrando que houve boa ressecção dos osteófitos. 


\section{Discussão}

A hiperostose esquelética idiopática difusa é uma doença degenerativa sistêmica que tem sido descrita com uma variedade de termos, incluindo doença de Forestier, espondilite ossificante ligamentosa, espondilite hiperostótica e hiperostose anquilosante da coluna ${ }^{3,4,7,8,16,35}$.

Em 1950, Forestier e Rotes-Querol ${ }^{8}$ descreveram, primeiramente, essa doença, denominando-a de hiperostose anquilosante senil.

Em 1970, Resnick ${ }^{25}$ propôs o termo hiperostose esquelética idiopática difusa para a doença de Forestier, chamando a atenção para a ossificação extra-espinhal e estabelecendo os critérios radiológicos. Esses critérios são os seguintes:

1 - Calcificação e ossificação da parede ânterolateral de quatro corpos vertebrais contíguos;

2 - Relativa preservação da altura discal dos espaços afetados;

3 - Ausência de anquilose na articulação sacroilíaca e apofisária.

Na doença de Forestier, os pacientes são, na maioria das vezes, assintomáticos, mas a doença pode se manifestar por um quadro doloroso raquiano, certa limitação dos movimentos da coluna, sem rigidez, ou com quadro álgico extra-espinhal (ombro, joelho) e com tendência à calcificação nos ligamentos e nos tendões, associada com dor nas articulações em um terço dos $\operatorname{casos}^{26}$.

A anormalidade radiológica característica da doença é a calcificação e a ossificação do ligamento longitudinal anterior da coluna. Esta é mais comumente visível na coluna torácica média, seguida da cervical e da lombar.

A disfagia é um sintoma encontrado em cerca de 17\% a $28 \%$ dos casos e requer tratamento cirúrgico em apenas $8 \%$ das vezes ${ }^{26}$. A patogênese da disfagia causada pelos osteófitos cervicais ainda não está bem definida. Alguns autores acreditam tratar-se de um fator provavelmente mecânico ${ }^{12}$, outros sugerem ser decorrente de uma reação do tipo inflamatória e edema pré-vertebral com esofagite e faringite, induzindo a uma fibrose e aderências ${ }^{2}$.

Oga e cols. ${ }^{20}$ acompanharam um paciente durante o período de 1982 a 1991. Inicialmente, o paciente apresentava apenas cervicalgia e hiperostose de $\mathrm{C} 3$ a C6; houve progressão dos osteófitos e da ossificação do ligamento longitudinal posterior, e a disfagia apareceu como sintomatologia 4 anos após. Foi constatado, radiologicamente, um importante desenvolvimento dos osteófitos localizados em C4-C5. Foi, então, submetido à ressecção do osteófito localizado nesse nível. Os autores concluíram que, sendo o esôfago fixado à cartilagem cricóide e ao diafragma, uma ossificação da região cervical em C4-C5 - que corresponde ao segmento móvel neste nível-, associada à presença de osteófitos, provoca a compressão do esôfago, causando disfagia ${ }^{20}$.
O nível cervical mais acometido na doença de Forestier é o C5-C6 (40\% dos casos), seguido de C4-C5 (23\%), C2-C3 e C3-C4 (14\% cada) $)^{18}$.

A fisiopatologia da doença é desconhecida. Alterações da elasticidade e da osteogênese, associadas a fatores locais, têm sido propostas, assim como o trauma local e alterações endócrinas ${ }^{18,34}$.

O diagnóstico diferencial da doença de Forestier inclui, principalmente, a espondilite deformante, a espondilite anquilosante e a osteocondrose intervertebral $^{5,8,25,34,35}$ (Tabela 1).

$\mathrm{O}$ tratamento adequado para a disfagia na doença de Forestier ainda é tema de controvérsias. Modificações na dieta e antiinflamatórios têm sido propostos $^{11,14,29}$. Radioterapia foi proposta, em 1951, por Piquet ${ }^{23}$, mas não foi adotada por outros autores. Contudo, o procedimento de escolha mais prudente no tratamento da disfagia provocada por essa afecção é a ressecção cirúrgica dos osteófitos. Alguns autores incluem a fusão, acreditando que, assim, a recorrência dos osteófitos seja menor ${ }^{13}$. Na literatura encontramos relatos de recorrência dos osteófitos 8 anos e 11 anos após a cirurgia ${ }^{13,31}$. Acreditamos que não seja necessária a utilização de enxertos ósseos em virtude da faixa etária que é acometida por essa doença, com pequena possibilidade tardia de recorrência.

Pouca melhora da disfagia é verificada após a cirurgia em casos de compressão crônica do esôfago, provavelmente em virtude de fibrose na parede esofágica. Daí a necessidade da intervenção mais precoce nos pacientes com sintomas leves ou médios, pois nesses casos os resultados são melhores.

Em casos de doença de Forestier, se não houver disfagia, mesmo a presença de importante calcificação do ligamento longitudinal posterior, não está indicada a sua ressecção, em virtude da dificuldade técnica dessa exérese, que pode vir acompanhada de alta morbidade. Poderá ser aventada a possibilidade de uma abordagem posterior, como uma laminoplastia ou laminectomia, de menor morbidade, em casos de compressão medular.

\section{Conclusão}

A doença de Forestier, ou hiperostose esquelética idiopática difusa, é caracterizada por ossificação importante do ligamento longitudinal posterior, encontrada principalmente em homens após a quinta década de vida.

A grande maioria dos pacientes é assintomática, mas pode ocorrer disfagia, que necessita de um tratamento cirúrgico. Este caso demonstra que o tratamento cirúrgico dos osteófitos por uma abordagem meticulosa anterior pode vir acompanhada de resultado gratificante. 


\begin{tabular}{|c|c|c|c|c|}
\hline \multirow[b]{2}{*}{$\mathbf{R x}$} & \multirow[b]{2}{*}{ Forestier } & \multirow{2}{*}{$\begin{array}{c}\text { Tabela } 1 \\
\text { Diagnóstico diferencial } \\
\text { Espondilite } \\
\text { anquilosante }\end{array}$} & & \multirow[b]{2}{*}{$\begin{array}{l}\text { Osteocondrose } \\
\text { intervertebral }\end{array}$} \\
\hline & & & $\begin{array}{l}\text { Espondilite } \\
\text { deformante }\end{array}$ & \\
\hline Disco intervertebral & Normal & Normal ou convexo & $\begin{array}{l}\text { Normal ou } \\
\text { altura diminuída }\end{array}$ & $\begin{array}{l}\text { Diminuição da } \\
\text { altura; fenômeno do vácuo }\end{array}$ \\
\hline Corpo vertebral & $\begin{array}{l}\text { Ossificação envolvendo } \\
\text { quatro corpos contíguos; } \\
\text { osteófitos largos }\end{array}$ & $\begin{array}{c}\text { Osteíte; } \\
\text { Extensa anquilose }\end{array}$ & $\begin{array}{c}\text { Osteófitos } \\
\text { anteriores largos }\end{array}$ & $\begin{array}{l}\text { Nódulos Schmoul; } \\
\text { Esclerose superior }\end{array}$ \\
\hline Esqueleto periférico & $\begin{array}{c}\text { Ossificação nas } \\
\text { articulações dos tendões }\end{array}$ & Normal & Normal & Normal \\
\hline Junção sacroilíaca & $\begin{array}{c}\text { Osteófitos } \\
\text { paraarticulares }\end{array}$ & $\begin{array}{l}\text { Erosão; Esclerose; } \\
\text { Anquilose óssea }\end{array}$ & Normal & Normal \\
\hline Junção apofisária & $\begin{array}{l}\text { Normal ou discreta } \\
\text { esclerose }\end{array}$ & $\begin{array}{l}\text { Erosão; Esclerose; } \\
\text { Anquilose óssea }\end{array}$ & Normal & $\begin{array}{l}\text { Alterações secundárias } \\
\text { à osteoartrite }\end{array}$ \\
\hline \multicolumn{5}{|l|}{ Clínica } \\
\hline Rigidez e dor na coluna & Comum & Severa & Pouco & Comum ou severa \\
\hline Etiologia & $\begin{array}{c}\text { Antígeno HLA B27 + } \\
\text { em } 34 \%\end{array}$ & $\begin{array}{c}\text { Antígeno HLA B27 + } \\
\text { em } 88 \% \text { a } 95 \%\end{array}$ & - & $\begin{array}{l}\text { Perda progressiva } \\
\text { de sulfato condroitina }\end{array}$ \\
\hline Incidência & $\begin{array}{c}6 \% \text { a } 12 \% \text { em } \\
\text { autópsia; mais comum no } \\
\text { sexo masculino; idade } \\
\text { média } 65 \text { a } 67 \text { anos }\end{array}$ & $\begin{array}{c}1 \% \text { a } 3 \% \text { em } \\
\text { homens jovens }\end{array}$ & $60 \%$ feminino & $\begin{array}{c}100 \% \text { acima dos } \\
90 \text { anos }\end{array}$ \\
\hline VHS & $\begin{array}{l}\text { Levemente elevada } \\
22 \% \text { dos casos }\end{array}$ & Elevada em $80 \%$ & Normal & Normal \\
\hline
\end{tabular}

\section{Referências}

1. BABORES M, FINNERTY JP: Aspiration preumonia secondary to giant cervical osteophyte formation (diffuse idiopathic skeletal hyperostosis or Forestier's disease): a case report. Chest 114:1481-2, 1998.

2. BAYER F: Dysphagia due to cervical spondylosis. J Laryngol Otol 67:615-30, 1953.

3. BICK E: Vertebral oteophytosis. A clinical syndrome. JAMA 160:828-9, 1956.

4. BICK E: Vertebral osteophytosis. Pathologic basis of its roentgenology. Am J Roentgenol Radium Theu Nucl Med 73:979-83, 1955.

5. CARLSON MJ, STAUFFER RN, PAYNE WS: Ankylosing vertebral hiperostosis causing dysphagia. Arch Surg 109:567-70, 1974.

6. FOGLIA FM, NOGUES OJ, GONZALES CX, ARIAS C: Dysphagia and estridor due to osteophytes of the cervical spine. Acta Otorrinolaringol Esp 49:71-4, 1998.

7. FORESTIER J, CAGIER R: Ankylosing hiperostosis of the spine: Clin Orthop Res 74:65-83, 1971.

8. FORESTIER J, ROTES-QUEROL J: Senile ankylosing hyperostosis of the spine. Ann Rheum Dis 321-30, 1950.

9. GAMACHE FW, VOORHIES RM: Hypertrophic cervical osteophytos causing disphagia. A review. J Neurosurg 53:338-44, 1980.

10. GRIFFITHS ID: Cervical mielopathy, ossification of the posterior longitudinal ligament and difuse idiopatic skeletal hyperostosis. Ann Rheum Dis 46: 116-68, 1988.

11. HARGROVE MD Jr: Dyphagia associated with inflamatory reaction within the esophagus at the level of a vertebral spur. Gastroint Endosc 13:28-9, 1966.

12. HECK CV: Hoarsenes and painful deglutition due to massive cervical exostoses. Surg. Gynecol Obstet 102:657-662, 1956.
13. HIRANO H, SUZUKI H, SAKAKIBARA T et al.: Dhysphagia due to hypertrophic cervical osteophytes. Clin Orthop 167:168-72, 1982.

14. LAMBERT JR, TEPPERMAN PS, JIMENEZ $\mathrm{J}$ et al.: Cervical spine disease and dysphagia. Four new cases and a review of the literature. Am J Gastroenterol 76:35-40, 1981.

15. LEROUX JL, LEGERON P, MOULINIOU L, LAROCHE M, MAZIERES B, BLOTMAN $F$ et al.: Stenosis of the lombar spinal canal in vertebral ankylosing hyperostosis Spine 17(10):1213-8, 1992.

16. MARAN A, JOCOBSON I: Cervical osteophyto preventing with pharyngeal symptoms. Laryngoscope 81:412-7, 1971.

17. MARKS B, SCHOBER E, SWOBODA H: Diffuse idiopathic skeletal hyperostosis causing obstructing laryngeal edema. Eur Arch Otorhinolaryngol 255(5):256-8, 1998.

18. MC CAFFERTY RR, HARRISON MJ, TOMAS LB, LARKINS MV: Ossification of the anterior longitudinal ligament and Forestier's disease: an analysis of seven cases. J Neurosurg 83:13-7, 1995.

19. MIZUNO J, NAKAGAWA H, ISOBE M: Dysphagia caused by ossification of the anteroir longitudinal ligament associated with diffuse idiopathic skeletal hyperostosis: report os two cases. No Shinkei Geka; 26(1):67-72, 1998.

20. OGA M, MASHIMA T, IWAKUMA T, SUGIOKA Y: Disphagia complications in ankylosing Spinal hyperestosis and ossification of the posterior longitudinal ligament. Spine 18:391-4, 1993.

21. PAPAKOSTAS K, THAKAU A, NANDAPALAN $V$, O'SULLIVAN G: An unusual case of stridor due to osteophytes of the cervical spine: (Forestier's disease). J Laryngol Otol Jan 113(1):65-7, 1999.

22. PAPPONE N, DI GIROLANO C, DEL PUENTE A, SCARPA R, ORIENTE P: Diffuse idiopathic skeletal 
hyperestosis (DISH): a retrospective analysis. Clin Rheumatol 15(2):121-4, 1996.

23. PIQUET J: Les troubles pharingolarynge's par spondylite deformante de la colunne cervical. Ann Otolaryngol 68:697-704, 1951.

24. REISNER A, STILES RG, TINDALL SC: Diffuse idiopathic skeletal hyperostosis causing acute thoracic myelopathy: a case report and discussion. Neuroscugery 26(3):507-11, 1990.

25. RESNICK D: Radiographic and pathologic feactures of spinal involvement to diffuse idiopathic skeletal hyperostosis. Radiology 119:559-68, 1976.

26. RESNICK D, SHAPIRO RF: Diffuse idiopathic skeletal hyperostosis (Ankylosing hyperostosis of Forestier and Rotes-Querol). Semin Artritis Rheumat 7:153-87, 1978.

27. RIVIEREZ M: Maladie de Forestier avec disfhagie. A propor d'un cas. Neurochirurgie 43(3):169-72, 1997.

28. ROLLAND Y, DROMER C, BENAZET JF, FOURNIE B: Hernie discale dorsale compressive responsable d'un syndrome de Brow - Sequard au cours d'une Maladie de Forestier. A propos d'un cas. Rachis 9:137-9, 1997.

29. SAUNDERS WH: Cervical osteophytes and dysphagia. Ann Otol Rhinol Laryngol 79:1091-7, 1970.

30. SILVA J, COSTA AOSM, BRAZÃO ES: Doença de Forestier. Relato de caso. Mini Conferência apresentada no VII Congresso da Sociedade Brasileira de Patologia da Coluna Vertebral. Comandatuba, Bahia, 22-25 de setembro, 1999.
31. SUZUKI K, ISHIDA Y, OHMORI K: Long term followup to diffuse idiopathic skeletal hiperostosis in the cervical spine. Analysis of progression of 35 . ossification. Neuroradiology 33:427-31, 1991.

32. TOLLEFSEN I, HOGNESTAD S, SUIHUS R: Forestier syndrome. A rare cause of dysphagia and dyspnea. Tidsskr Nor Laegeforem 20:117(28):4076-8, 1997.

33. TSUKAMOTO Y, ONITSUKA H, LEE K: Radiologic aspects of diffuse idiopathic skeletal hyperostosis in the spine. Am J Roentgenology 129:913-8, 1977.

34. UTSINGER PP, RESNICK D, SHAPIRO R: Diffuse skeletal abnormalities in Forestier disease. Arch Intern Med 136:763-8, 1976.

35. VERNON RB, PIRIE CJ, TRENWITH V: Pathology of the dorsal spine in ankylosing hyperostosis. Ann Rheum Dis 33:281-8, 1974.

Original recebido em maio de 2000

Aceito para publicação em agosto de 2001

\section{Endereço para correspondência:}

Fernando Luíz Rolemberg Dantas

Rua Muzambinho, 458 - ap. 1.201

CEP 30210-530 - Belo Horizonte, $M G$ 